\title{
DETERMINING THE MASSES AND EVOLUTION OF CVS THROUGH ELLIPSOIDAL VARIATIONS
}

\author{
Heather L. Osborne, ${ }^{1}$ Thomas E. Harrison, ${ }^{1}$ Joni J. Johnson, ${ }^{1}$ and Steve B. Howell ${ }^{2}$
}

We use simultaneous multi-wavelength $B V R I$ $J H K$ photometric observations of cataclysmic variables (CVs) to determine the inclination angles of the systems, together with phaseresolved spectroscopy to calculate the $K_{2}$ and isini. We then calculate the masses of the system components. We are using these data to construct mass-radius diagrams of the secondaries in an effort to resolve some of the debate over their evolutionary history.

Our understanding of $\mathrm{CV}$ evolution is being called into question (for example see Harrison et. al. 2004 , this proceedings). Howell et. al (2001, hereafter HNR.) provide predictions for a mass-radius relationship of secondaries based on currently accepted evolutionary theory. The MI-R diagram for secondaries should show them to be larger for their radii than main-sequence stars. By experimentally determining the masses of a set of secondaries, we can create such a mass-radius diagram and test the favored paradigm. We require the inclination angle $i$, $v \sin i$ and semi-amplitude of the radial velocity curve $\mathrm{K}_{2}$ to obtain these masses. We use phase-resolved $B I R I J H K$ observations of $\mathrm{CVs}$ to construct light curves from which we extract inclination angles using Wilson-Devinney '98. Phase-resolved spectroscopy using the sodium doublet at 8190.4 allows us to construct radial velocity curves from which we can obtain the $\mathrm{K}_{2}$ and $2 \sin i$.

The infrared photometry presented here was all obtained with KPNO's $2.1 \mathrm{~m}$ with SQIID over the summer, the spectroscopy from APO $3.5 \mathrm{~m}$ with DIS in September. JHK bands are used to resolve the ellipsoidal variations because the secondary star is the dominant contributor of flux in the infrared; the sodium doublet at $8190 A$ is used for the same reasons. We here present a light curve and best-fit model for AM Her, and a radial velocity curre for IP Peg, as examples of our current work. AN Her was found to have an inclination angle of $59 \pm 3$ degrees. We require further data for IP Peg in the 0.4 to 0.7 orbital phase range to properly constrain the model shown here, but the preliminary results are in good

\footnotetext{
${ }^{1}$ Department of Astronomy, New Mexico State Iniversity, Las Cruces, NM.

${ }^{2}$ National Optical Astronomical (Observatory
}

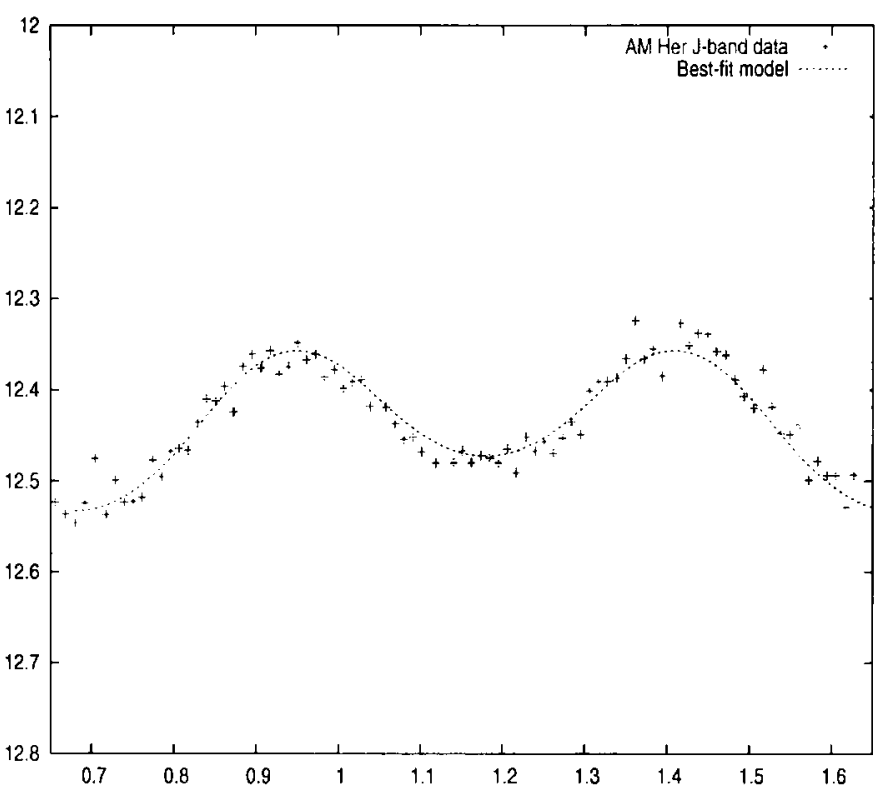

Fig. 1. J-band data for AM Her (unconnected points), together with the best-fit model (line).

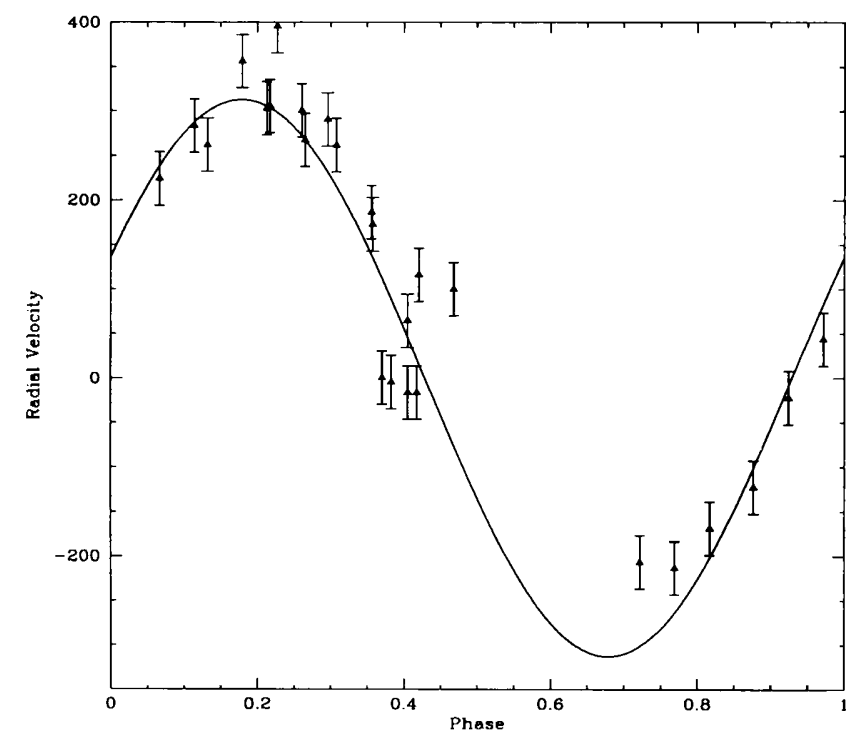

Fig. 2. Radial velocity curve for IP Peg (triangles), together with the best-fit sine wave (line).

accord with the published value of $288 \mathrm{~km} / \mathrm{sec}$.

\section{REFERENCES}

Harrison, T.E., et. al., 2004, this proceedings

Howell, S.B., Nelson, L.A., \& Rappaport, S. 2001, ApJ, 550,89 